\title{
On the Minimum Span of Cone, Tadpole, and Barbell Graphs
}

\author{
Hafif Komarullah, Ikhsanul Halikin*, Kiswara Agung Santoso \\ Graph, Combinatorics, and Algebra Research Group, Department of Mathematics, FMIPA, University of Jember \\ *Corresponding author. Email: ikhsan.fmipa@unej.ac.id
}

\begin{abstract}
Let $G$ be a simple and connected graph with $p$ vertices and $q$ edges. An $L(2,1)$-labelling on the graph $G$ is a function $f: V(G) \rightarrow\{0,1, \ldots, k\}$ such that every two vertices with a distance one receive labels that differ by at least two, and every two vertices at distance two receive labels that differ by at least one. A number $k$ is called as span of $L(2.1)$ labelling, if $k$ is the largest vertex labels. The span of a graph $G$ can be more than one, the minimum value of the span of a graph $G$ is notated by $\lambda_{(2,1)}(G)$. In this paper, we determine the minimum span of cone, tadpole, and barbell graphs
\end{abstract}

Keywords: L(2,1)-labelling, Minimum of span, Cone, Tadpole, and Barbell graphs.

properties about $L(2,1)$-labelling which will be used in this paper as follows.

\section{INTRODUCTION}

Throughout this paper, all graphs are simple, connected, and undirected. Graph labelling is one of the research topic in graph theory. Graph labelling was first introduced in the mid-1960s. There are many kind research about graph labelling, one of them is $L(2,1)$ labelling. An $L(2,1)$-labelling is defined as a mapping from the set of vertices in graph to a set of non-negative integer such that the absolute value of difference between the vertex labels with distance one is two and the absolute value of difference between the vertex labels with distance two is one [1]. Formally, let $G=(V, E)$ be a graph and $d(u, v)$ notated the distance between vertex $u$ and $v$. a function $f: V(G) \rightarrow\{0,1, \ldots, k\}$ is called $L(2,1)$ labelling if $|f(u)-f(v)| \geq 1$ for $d(u, v)=2$ and $|f(u)-f(v)| \geq 2$ for $d(u, v)=1$. The number $k$ here is called as span of $L(2.1)$-labelling if $k$ is the largest vertex labels. The span of a graph $G$ can be more than one, the minimum value of the span of a graph $G$ is notated by $\lambda_{(2,1)}(G)[2,3]$.

There are many research about the minimum span of a graph. Griggs and Yeh [3,4] in 1992 proved that $\lambda_{2,1}\left(S_{1, n}\right)=n+1, \lambda_{2,1}\left(C_{n}\right)=4$ and $\lambda_{2,1}\left(P_{n}\right)=4$. The minimum span of fan graph $\left(f_{n}\right)$ is $n+1$ and wheel graph $\left(W_{n}\right)$ is $n+1$ [5]. Yuri et al. [6] in 2018 proved that the minimum span of Sierpinski graph $\left(S_{(n, m)}\right)$ is 4 for $m=2$ and $m=3$.

In this paper, we will discuss about the minimum span of cone, tadpole, and barbell graphs. There are some
Lemma 1.1 [7] If $H$ is a subgraf of graph $G$, then $\lambda_{2,1}(H) \leq \lambda_{2,1}(G)$

Lemma 1.2 [3] Let $C_{n}$ be a cycle graph with $n \geq 3$, then $\lambda_{2,1}\left(C_{n}\right)=4$

\section{MAIN RESULT}

In this section we will discuss about the minimal span of cone, tadpole, and barbell graphs

\subsection{Cone Graph}

Cone graph is a graph formed from a join operation between graph cycle $C_{m}$ and a graph $\bar{K}_{n}$. A cone graph is denoted by $C_{m, n}$. The set of vertices and edges on a cone graph will be notated as follows.

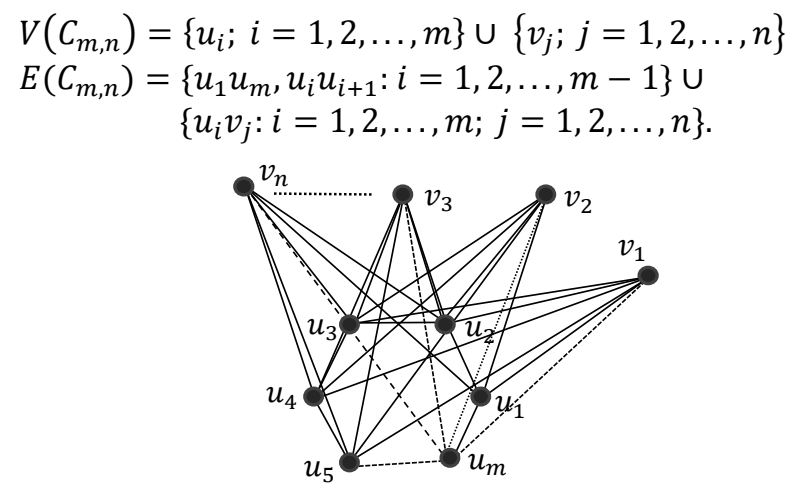

Figure 1 Cone graph $C_{m, n}$. 
Theorem 2.1 Let $C_{m, n}$ be a cone graph with $m \geq 3$ and $n \geq 1$, then $\lambda_{2,1}\left(C_{m, n}\right)=n+5$.

Proof. First, we will show that $\lambda_{2,1}\left(C_{m, n}\right) \geq n+5$. Suppose that cone graph $C_{m, n}$ can be labelled only with labels $0,1,2, \ldots, n+4$. Since cycle graph $C_{n}$ is a subgraph of cone graph $C_{m, n}$, then based on the Lemma 1.2, the largest label of vertices $u_{i}$ is 4 . As the distance of vertex $u_{i}$ and $v_{j}$ is one, then the absolute value of the difference between the labels is at least two. Therefore, vertices $v_{j}$ must be labelled by $6,7, \ldots, n+4$. Since the distance of vertex $v_{j}$ and $v_{j+1}$ is two, then the absolute value of the difference between the labels is at least one. Thus, every label of $v_{j}$ must be different. Since the number of vertices of $\left\{v_{j}\right\}$ is $n$, while the number of label used is $n-1$, then according to the pigeonhole principle, there are at least two vertices in $v_{j}$ that has the same label. So, the cone graph cannot be labelled only with labels $1,2, \ldots, n+4$. Therefore, we can conclude that $\lambda_{2,1}\left(C_{m, n}\right) \geq n+5$.

Next, we will show that $\lambda_{2,1}\left(C_{m, n}\right) \leq n+5$ by constructing the $L(2,1)$-labelling on a cone graph. Its labelling will be divided by three cases as follows.

1. for $m \equiv 0 \bmod 3$

$f\left(u_{i}\right)=\left\{\begin{array}{l}0, i \equiv 1 \bmod 3 \\ 2, i \equiv 2 \bmod 3 \\ 4, i \equiv 0 \bmod 3\end{array}\right.$

$f\left(v_{j}\right)=j+5 ; j=1,2, \ldots, n$

Based on the condition of $L(2,1)$-labelling, the absolute value of the difference between the labels for every two vertices with distance one is at least two. This will be shown as follows.

a. The difference of vertex label $u_{i}$ and $u_{i+1}$ for $i \equiv$ $1 \bmod 3$

$\left|f\left(u_{i}\right)-f\left(u_{i+1}\right)\right|=|0-2| \geq 2$

b. The difference of vertex label $u_{i}$ and $u_{i+1}$ for $i \equiv$ $2 \bmod 3$

$\left|f\left(u_{i}\right)-f\left(u_{i+1}\right)\right|=|2-4| \geq 2$

c. The difference of vertex label $u_{i}$ and $u_{i+1}$ for $i \equiv$ $0 \bmod 3$

$\left|f\left(u_{i}\right)-f\left(u_{i+1}\right)\right|=|4-0| \geq 2$

d. The difference of vertex label $v_{j}$ and $u_{i}$ for $j=$ $1,2, \ldots, n$

$\left|f\left(v_{j}\right)-f\left(u_{i}\right)\right|=|j+5-0| \geq 2, i \equiv 1 \bmod 3$

$\left|f\left(v_{j}\right)-f\left(u_{i}\right)\right|=|j+5-2| \geq 2, i \equiv 2 \bmod 3$

$\left|f\left(v_{j}\right)-f\left(u_{i}\right)\right|=|j+5-4| \geq 2, i \equiv 0 \bmod 3$

In addition, according to the rule of $L(2,1)$-labelling, the absolute value of the difference between the labels for every two vertices with distance two is at least one. This will be shown as follows.

a. The difference of vertex label $u_{i}$ and $u_{i+2}$ for $i \equiv$ $1 \bmod 3$

$\left|f\left(u_{i}\right)-f\left(u_{i+2}\right)\right|=|0-4| \geq 1$

b. The difference of vertex label $u_{i}$ and $u_{i+2}$ for $i \equiv$ $2 \bmod 3$ $\left|f\left(u_{i}\right)-f\left(u_{i+2}\right)\right|=|2-0| \geq 1$

c. The difference of vertex label $u_{i}$ and $u_{i+2}$ for $i \equiv$ $0 \bmod 3$

$\left|f\left(u_{i}\right)-f\left(u_{i+2}\right)\right|=|4-2| \geq 1$

d. The difference of vertex label $v_{t}$ and $v_{s}$ for $t \neq$ $s, s \geq t+1$, dan $1 \leq t<s \leq j$

$$
\begin{aligned}
\left|f\left(v_{s}\right)-f\left(v_{t}\right)\right| & =|(s+5)-(t+5)|=|s-t| \\
& =|(t+1)-t| \geq 1
\end{aligned}
$$

We can conclude that for $m \equiv 0 \bmod 3$, the function $f$ satisfied $L(2,1)$-labelling.

2. for $m \equiv 1 \bmod 3$

$$
\begin{aligned}
& f\left(u_{i}\right)=\left\{\begin{array}{l}
0, i \equiv 1 \bmod 3 \text { dan } i \neq m \\
2, i \equiv 2 \bmod 3 \text { dan } i \neq m-2 \\
4, i \equiv 0 \bmod 3 \text { dan } i \neq m-1 \\
3, i=m-2 \\
1, i=m-1 \\
4, i=m
\end{array}\right. \\
& f\left(v_{j}\right)=j+5, j=1,2, \ldots, n
\end{aligned}
$$

In the same way with the Case 1 , we can prove that the function $f$ satisfied $L(2,1)$-labelling for $m \equiv 1 \bmod 3$.

3. for $m \equiv 2 \bmod 3$

$f\left(u_{i}\right)=\left\{\begin{array}{l}0, i \equiv 1 \bmod 3 \text { dan } i \neq m-1 \\ 2, i \equiv 2 \bmod 3 \text { dan } i \neq m \\ 4, i \equiv 0 \bmod 3 \\ 1, i=m-1 \\ 3, i=m\end{array}\right.$
$f\left(v_{j}\right)=j+5 ; j=1,2, \ldots, n$

Again, it is easy to prove that the function $f$ satisfied $L(2,1)$-labelling for $m \equiv 2 \bmod 3$. So, we have shown that $\lambda_{2,1}\left(C_{m, n}\right) \leq n+5$. Therefore, we can conclude that $\lambda_{2,1}\left(C_{m, n}\right)=n+5$. For example, an $L(2,1)$ labelling on a cone graph can be seen in Figure 2.

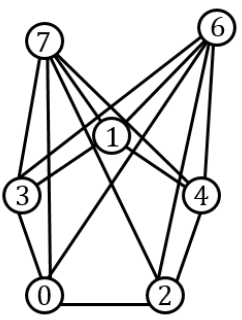

Figure $2 L(2,1)$-labelling of cone graph $C_{5,2}$.

\subsection{Tadpole Graph}

Tadpole graph $\left(T_{m, n}\right)$ is defined as a graph obtained by combining a vertex of cycle graph $C_{m}$ with one of the leaf of path graph $P_{n}$. Suppose that the vertices and edges in the tadpole graph are notated as follows.

$$
\begin{aligned}
& V\left(T_{m, n}\right)=\left\{u_{i} ; i=1,2, \ldots, m\right\} \cup\left\{v_{j} ; j=1,2, \ldots, n\right\} \\
& E\left(T_{m, n}\right)=\left\{u_{1} u_{m}, u_{i} u_{i+1} ; i=1,2, \ldots, m-1\right\} \cup \\
& \left\{u_{1} v_{1}\right\} \cup\left\{v_{i} v_{i+1} ; i=1,2, \ldots, n-1\right\}
\end{aligned}
$$




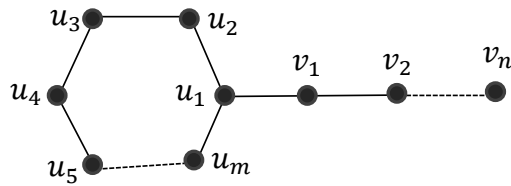

Figure 3 Tadpole graph $T_{m, n}$.

Theorem 2.2 Let $T_{m, n}$ be a tadpole graph with $m \geq 3$ and $n \geq 1$, then $\lambda_{2,1}\left(T_{m, n}\right)=4$.

Proof. Let $T_{m, n}$ be a tadpole graph with $m \geq 3$ and $n \geq$ 1 , We will prove that $\lambda_{2,1}\left(T_{m, n}\right) \geq 4$. Since the cycle graph $C_{m}$ is a subgraph of tadpole graph $T_{m, n}$, then based on the Lemma 1.2, we get $\lambda_{2,1}\left(T_{m, n}\right) \geq \lambda_{2,1}\left(C_{m}\right)=4$. So, we have proved that $\lambda_{2,1}\left(T_{m, n}\right) \geq 4$.

Next, we will show that $\lambda_{2,1}\left(T_{m, n}\right) \leq 4$ by constructing $L(2,1)$-labelling on the tadpole graph $T_{m, n}$. We will consider four cases as follows.

1. for $m=4$

$$
\begin{aligned}
& f\left(u_{i}\right)=\left\{\begin{array}{l}
0, i=1 \\
3, i=2 \\
1, i=3 \\
4, i=4
\end{array}\right. \\
& f\left(v_{j}\right)=\left\{\begin{array}{l}
2, j \equiv 1 \bmod 3 \\
4, j \equiv 2 \bmod 3 \\
0, j \equiv 0 \bmod 3
\end{array}\right.
\end{aligned}
$$

2. for $m \equiv 0 \bmod 3$

$$
\begin{aligned}
& f\left(u_{i}\right)=\left\{\begin{array}{l}
0, i \equiv 1 \bmod 3 \\
2, i \equiv 2 \bmod 3 \\
4, i \equiv 0 \bmod 3
\end{array}\right. \\
& f\left(v_{j}\right)=\left\{\begin{array}{l}
3, j \equiv 1 \bmod 4 \\
1, j \equiv 2 \bmod 4 \\
4, j \equiv 3 \bmod 4 \\
0, j \equiv 0 \bmod 4
\end{array}\right.
\end{aligned}
$$

3. for $m \equiv 1 \bmod 3$ and $m \neq 4$

$$
\begin{aligned}
& f\left(u_{i}\right)=\left\{\begin{array}{l}
0, i \equiv 1 \bmod 3 \text { and } i \neq m \\
2, i \equiv 2 \bmod 3 \text { and } i \neq m-2 \\
4, i \equiv 0 \bmod 3 \text { and } i \neq m-1 \\
3, i=m-2 \\
1, i=m-1 \\
4, i=m
\end{array}\right. \\
& f\left(v_{j}\right)=\left\{\begin{array}{l}
3, j \equiv 1 \bmod 4 \\
1, j \equiv 2 \bmod 4 \\
4, j \equiv 3 \bmod 4 \\
0, j \equiv 0 \bmod 4
\end{array}\right.
\end{aligned}
$$

4. for $m \equiv 2 \bmod 3$

$$
\begin{aligned}
& f\left(u_{i}\right)=\left\{\begin{array}{l}
0, i \equiv 1 \bmod 3 \text { dan } i \neq m-1 \\
2, i \equiv 2 \bmod 3 \text { dan } i \neq m \\
4, i \equiv 0 \bmod 3 \\
1, i=m-1 \\
3, i=m
\end{array}\right. \\
& f\left(v_{j}\right)=\left\{\begin{array}{l}
4, j \equiv 1 \bmod 3 \\
2, j \equiv 2 \bmod 3 \\
0, j \equiv 0 \bmod 3
\end{array}\right.
\end{aligned}
$$

In the same way as Theorem 2.1 , it is easy to prove that every two vertices with distance one receive labels that differ by at least two, and every two vertices at distance two receive labels that differ by at least one. So, we have proved that $\lambda_{2,1}\left(T_{m, n}\right) \leq 4$. Therefore, we can conclude that $\lambda_{2,1}\left(T_{m, n}\right)=4$. As illustration of Theorem 2.2, we present an example of $L(2,1)$-labeling on a tadpole graph in Figure 4.

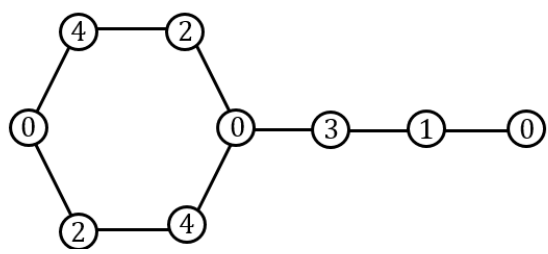

Figure $4 L(2,1)$-labelling of tadpole graph $T_{6,3}$.

\subsection{Barbell Graph}

Barbell graph $\left(B_{n}\right)$ is a graph obtained by connecting two complete graph $K_{n}$ by an edge. Suppose that the vertices and edges in the barbell graph are notated as follows. $V\left(B_{n}\right)=\left\{u_{i} ; i=1,2, \ldots, n\right\} \cup\left\{v_{j} ; j=1,2, \ldots, n\right\}$ $E\left(B_{n}\right)=\left\{u_{s} u_{t}, s \neq t\right\} \cup\left\{u_{1} v_{1}\right\} \cup\left\{v_{s} v_{t} ; s \neq t\right\}$

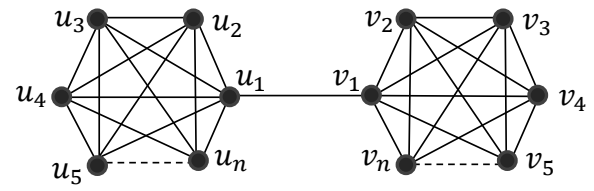

Figure 5 Barbell graph $B_{n}$.

Theorem 2.3 Let $B_{n}$ be a barbell graph with $n \geq 3$, then $\lambda_{2,1}\left(B_{n}\right)=2 n-1$.

Proof. First, we will prove that $\lambda_{2,1}\left(B_{n}\right) \geq 2 n-1$. Suppose that barbell graph $B_{\mathrm{n}}$ can be labelled by $0,1,2, \ldots, 2 n-2$. Since barbell graph formed by two complete graphs and every two vertices are adjacent, then based on the rule of $L(2,1)$-labelling, the absolute value of the different between the labels is at least two. Therefore, every complete graph in a barbell graph must be labelled by even number or odd number only. This shows that the barbell graph requires $n$ even labels and $n$ odd labels. But, there are just $n$ even labels and $n-1$ odd labels. Therefore, we need one odd label except $0,1,2, \ldots, 2 n-2$. So, it is obvious that $\lambda_{2,1}\left(B_{n}\right) \geq 2 n-$ 1.

Next, we will prove that $\lambda_{2,1}\left(B_{n}\right) \leq 2 n-1$ by constructing the $L(2,1)$-labelling on a barbell graph. Let $f: V\left(B_{n}\right) \rightarrow\{0,1,2, \ldots, 2 n-1\}$ be a function as follows. $f\left(u_{i}\right)=2 i-2, i=1,2, \ldots, n$

$f\left(v_{j}\right)=2(n-j)+1, j=1,2, \ldots, n$

It is easy to prove that $\lambda_{2,1}\left(B_{n}\right) \leq 2 n-1$. Therefore, we can conclude that $\lambda_{2,1}\left(B_{n}\right)=2 n-1$. In the figure 6 , we give an example of $L(2,1)$-labelling on barbell graph with $n=4$. 


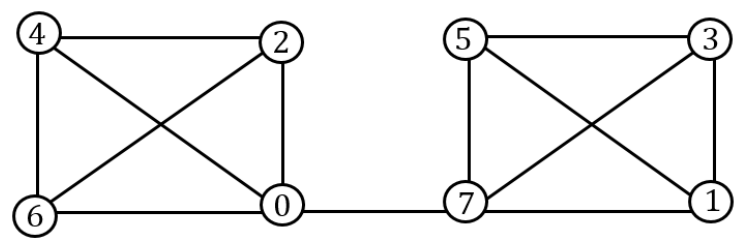

Figure $6 L(2,1)$-labelling of barbell graph $B_{4}$.

\section{ACKNOWLEDGMENTS}

This research has been supported by "Hibah Kelompok Riset Graf, Combinatorika, dan Aljabar (GCA), Universitas Jember, Tahun Anggaran 2021, Nomor 14737/UN25/LT/2021"

\section{REFERENCES}

[1] P. Bella, D. Král, B. Mohar, K. Quittnerová, Labeling planar graphs with a condition at distance two, European Journal of Combinatorics, vol. 28, Issue 8, 2007, Pages 2201-2239. DOI: https://doi.org/10.1016/j.ejc.2007.04.019
[2] Z. Shao, R.K. Yeh, D. Zhang, The (2, 1)-Labeling on Graphs and Frequency Assigment Problem. Applied Mathematics Letters, vol. 21, 2008, pp.3741. DOI: https://doi.org/10.1016/j.aml.2006.08.029

[3] J. Griggs, R. Yeh, Labelling graph with a condition at distance 2, SIAM J. Discrete Math, vol. 5, 1992, pp. 586-595. DOI: https://doi.org/10.1137/0405048

[4] R.K. Yeh, A survey on labeling graphs with a condition at distance two, Discrete Mathematics, vol. 306, Issue 12, 2006, pp. 1217-1231. DOI: https://doi.org/10.1016/j.disc.2005.11.029

[5] S. Fatimah, I.W. Sudarsana, S. Musdalifah, Pelabelan L(2,1) Pada Operasi Beberapa Kelas Graf (in Indonesian), Jurnal Ilmiah Matematika dan Terapan, 2016, 13(2), pp. 73-84.

[6] C. S. Yuri, Susiana, Pelabelan L $(2,1)$ pada Graf Sierpinski $S(n, k)$ (in Indonesian), Jurnal Sains Indonesia, 2018, 42(1), pp. 22-24. DOI: https://doi.org/10.24114/ jsi.v42i1.12244

[7] A. Lum, Upper Bound on L(2,1)-labelling Number of Graphs with Maximum Degree $\Delta, 2007$, https://www.whitman.edu/documents/academics/m athematics/lumaa.pdf 